\title{
Synthesis, Characterization and Biological Evaluation of Some Novel Thiophene Anchored Fluorinated Heterocycles
}

\author{
B. K. KARALE ${ }^{1 *}$, H. N. AKOLKAR ${ }^{1}$, A. S. BURUNGALE ${ }^{2}$, \\ S. D. MHASKE ${ }^{1}$ and R. S. ENDAIT ${ }^{1}$
}

\begin{abstract}
'Department of Chemistry, Radhabai Kale Mahila Mahavidyalaya, Ahmednagar - 414001, India. ${ }^{2}$ Post-Graduate and Research Center in Chemistry, S. M. Joshi College, Hadapsar, Pune-28, India. ${ }^{*}$ Corresponding author Email: bkkarale@yahoo.com
\end{abstract}

http://dx.doi.org/10.13005/ojc/310155

(Received: December 03, 2014; Accepted: January 10, 2015)

\begin{abstract}
A new series of thiophene anchored 1,3,4-thiadiazole, 1,2,4-triazole, 2-heteryl chromone, 1,5-benzothiazepine, pyrazoline, 2-styryl chromone derivatives containing fluorine are synthesized, characterized by spectral methods and screened them for various biological activities.
\end{abstract}

Key words: 1,3,4-Thiadiazole, 1,2,4-Triazole, 2-Heteryl chromone, 1,5-Benzothiazepine, Pyrazoline, 2-Styryl chromone.

\section{INTRODUCTION}

Thiophene is sulfur containing five membered heterocyclic compound widely used as building block in agrochemicals ${ }^{1}$. Thiophene containing compounds exhibit antimicrobial ${ }^{2}$, antiparasitic ${ }^{3}$, anticancer ${ }^{4}$ and anticonvulsant ${ }^{5}$ activities.

2-Styryl chromones are associated with various pharmacological activities such as antiallergic ${ }^{6}$, cytotoxic ${ }^{7}$, antioxidant ${ }^{8}$, antiinflammatory $^{9}$ and antibacterial ${ }^{10}$. 2-Styryl chromones are also acts as ${ }^{2}$-amyloid imaging agents ${ }^{11}$. Pyrazole derivatives are known for various potent biological activities such as antibacterial ${ }^{12}$, antioxidant ${ }^{12}$, anticancer ${ }^{13}$, ACE inhibitor ${ }^{14}$.

Thiadiazole derivatives possess antitumor ${ }^{15}$, antiinflammatory ${ }^{16}$, SIRT1 inhibitor ${ }^{17}$, antihypertensive ${ }^{18}$, antimicrobial ${ }^{19}$ activities. 1,2,4Triazole anchored compounds are associated with antimicrobial ${ }^{19}$, antioxidant ${ }^{20}$, anti-inflammatory ${ }^{20}$, CYP enzyme inhibitor ${ }^{21}$, anxiolytic ${ }^{22}$ activities.

Compounds containing chromone scaffold are acts as breast cancer resistance protein ABCG2 inhibitor ${ }^{23}$, monoamine oxidase inhibitor ${ }^{24}$, adenosine $A_{2 A}$ receptor antagonists ${ }^{25}$. Some 1,5benzothiazepines are antifungal ${ }^{26}$, 
anticonvulsant ${ }^{27}$, anti breast cancer ${ }^{28}$, antithrombotic ${ }^{29}$, antidepressant ${ }^{30}$ agents. Compounds having pyrazoline moity are known to possess anti-inflammatory ${ }^{31}$, antimalarial ${ }^{32}$, antitubercular ${ }^{33}$, antidepressant ${ }^{34}$ activities.

The activities associated with these various heterocycles prompted us to synthesize some novel thiophene anchored fluorinated heterocycles.

\section{Biological activities}

Antimicrobial activity

Synthesized compounds were screened for their antifungal and antibacterial activities. The in vitro antimicrobial activities of the synthesized compounds were assessed against fungi and bacteria. The fungi used were $C$. albicans, $A$. Fumigatus and $A$. Niger. The bacterias used were S. aureus, E. coli, S. Epidermidis and P. Vulgaris.

Fluconazole and Amikacin were used as standards for comparison for antifungal and antibacterial activities respectively. The activities were determined by measuring the diameter of the inhibition zone in $\mathrm{mm}$.

None of the compounds is a suitable candidate for antifungal and antibacterial indication as shown in Table 2.

\section{EXPERIMENTAL}

Melting points were recorded in open capillaries in liquid paraffin bath and are uncorrected. IR spectra were recorded on PerkinElmer FTIR spectrophotometer in $\mathrm{KBr}$ disc. ${ }^{1} \mathrm{H}$ NMR spectra were recorded on Bruker $400 \mathrm{MHz}$ NMR spectrometer in DMSO as a solvent and TMS as an internal standard. Peak values are shown in ' (ppm). Mass spectra were recorded on Finnigan mass spectrometer.

2-\{[4-Bromo-5-(methylsulfanyl)thiophen-2yl]carbonyl\}-N-phenylhydrazinecarbothioamide 2 Equimolar amounts $(0.01$ mole) of compound 1 and aryl isothiocyanate were dissolved in $15 \mathrm{~mL}$ of ethanol. The reaction mixture was heated under reflux for 55 minutes. The progress of reaction was monitored by TLC. After completion of reaction the contents were cooled and the solid obtained was filtered and crystallized from ethanol to get compound 2.

$2 \mathbf{a}$

IR $\left(\mathrm{KBr}, \mathrm{cm}^{-1}\right): 3327,3228,3113,1643$, 1257; ${ }^{1} \mathrm{H}$ NMR (DMSO): $\delta 2.22$ (s, 3H), 3.3 (s, 3H), 7.00-7.20 (m, 4H), $7.8(\mathrm{~s}, 1 \mathrm{H}), 9.5(\mathrm{~s}, 2 \mathrm{H}), 10.6$ (s, $1 \mathrm{H})$; MS: $\mathrm{m} / \mathrm{z} 430\left(\mathrm{M}^{+}\right)$; Elemental Anal. Calcd.: C, 38.89; H, 3.26; N, 9.72; found: C, 38.91; H, 3.29; N, $9.74 \%$.

$2 b$

IR $\left(\mathrm{KBr}, \mathrm{cm}^{-1}\right): 3324,3220,3117,1640$, 1262; ${ }^{H} \mathrm{H}$ NMR (DMSO): $\delta 2.21$ (s, 3H), 7.04-7.21 (m, 4H), 7.87 (s, 1H), 9.54 (s, 2H), 10.64 (s, 1H); MS: m/ z $418\left(\mathrm{M}^{+}\right)$; Elemental Anal. Calcd.: C, 37.15; H, 2.64; N, 10.00; found: C, 37.18; H, 2.67; N, $10.04 \%$.

2c

IR $\left(\mathrm{KBr}, \mathrm{cm}^{-1}\right): 3330,3224,3109,1647$, 1255; ${ }^{~} \mathrm{H}$ NMR (DMSO): $\delta 2.21$ (s, 3H), 7.03-7.20 (m, 4H), 7.86 (s, 1H), 9.53 (s, 2H), 10.62 (s, 1H); MS: m/ z $434\left(\mathrm{M}^{+}\right)$; Elemental Anal. Calcd.: C, 35.75; H, 2.54; N, 9.62; found: C, 35.78; H, 2.57; N, $9.65 \%$.

2d

IR $\left(\mathrm{KBr}, \mathrm{cm}^{-1}\right): 3328,3225,3111,1641$, 1257; ${ }^{1} \mathrm{H}$ NMR (DMSO): $\delta 2.22$ (s, 3H), 7.00-7.25 (m, $5 \mathrm{H}), 7.83(\mathrm{~s}, 1 \mathrm{H}), 9.52$ (s, 2H), 10.65 (s, 1H); MS: m/ z $400\left(\mathrm{M}^{+}\right)$; Elemental Anal. Calcd.: C, 38.81; H, 3.01; N, 10.44; found: C, 38.84; H, 3.05; N, $10.48 \%$.

$2 e$

IR $\left(\mathrm{KBr}, \mathrm{cm}^{-1}\right): 3330,3224,3119,1651$, 1264; ${ }^{1} \mathrm{H}$ NMR (DMSO): $\delta 2.20$ (s, 3H), 3.34 (s, 3H), 7.05-7.24 (m, 4H), $7.81(\mathrm{~s}, 1 \mathrm{H}), 9.53(\mathrm{~s}, 2 \mathrm{H}), 11.01$ (s, 1H); MS: m/z $430\left(\mathrm{M}^{+}\right)$; Elemental Anal. Calcd.: C, 38.89; H, 3.26; N, 9.72; found: C, 38.92; H, 3.29; N, $9.74 \%$.

5-(4-Bromo-5-(methylthio)thiophen-2-yl)-Nphenyl-1,3,4-thiadiazol-2-amine 3

Thiosemicarbazide 2 (0.001 mole) was dissolved in $3 \mathrm{~mL}$ of conc. $\mathrm{H}_{2} \mathrm{SO}_{4}$ in $50 \mathrm{~mL}$ beaker. The reaction mixture was stirred at room temperature for $3 \mathrm{hr}$. After completion of reaction 10 $\mathrm{g}$ of crushed ice was added in it. The solid obtained was separated by filtration and crystallized from 1:1 mixture of DMF and water to afford thiadiazole 3. 
$3 a$

IR $\left(\mathrm{KBr}, \mathrm{cm}^{-1}\right): 3161,1633,1591 ;{ }^{1} \mathrm{H}$ NMR (DMSO): $\delta 2.3(\mathrm{~s}, 3 \mathrm{H}), 3.5(\mathrm{~s}, 3 \mathrm{H}), 6.9-7.4(\mathrm{~m}, 4 \mathrm{H})$, 7.8 (s, 1H), 10.9 (s, 1H); MS: m/z $412\left(\mathrm{M}^{+}\right)$; Anal. Calcd.: C, 40.58; H, 2.92; N, 10.14; found: C, 40.60; $\mathrm{H}, 2.94 ; \mathrm{N}, 10.17 \%$.

\section{$3 b$}

IR $\left(\mathrm{KBr}, \mathrm{cm}^{-1}\right): 3166,1630,1587 ;{ }^{1} \mathrm{H}$ NMR (DMSO): $\delta 2.31$ (s, 3H), 6.95-7.82 (m, 5H), 10.94 (s, $1 \mathrm{H})$; MS: m/z $400\left(\mathrm{M}^{+}\right)$; Anal. Calcd.: C, 38.81; H, 2.25; N, 10.44; found: C, 38.84; H, 2.28; N, 10.47\%.

$3 c$

IR $\left(\mathrm{KBr}, \mathrm{cm}^{-1}\right): 3156,1638,1598 ;{ }^{1} \mathrm{H}$ NMR (DMSO): $\delta 2.30$ (s, 3H), 6.91-7.83 (m, 5H), 10.91 (s, 1H); MS: m/z $416\left(\mathrm{M}^{+}\right)$; Anal. Calcd.: C, 37.28; H, 2.17; N, 10.03; found: C, 37.31; H, 2.20; N, $10.06 \%$.

\section{$3 d$}

IR $\left(\mathrm{KBr}, \mathrm{cm}^{-1}\right): 3167,1632,1588 ;{ }^{1} \mathrm{H}$ NMR (DMSO): $\delta 2.31$ (s, 3H), 6.92-7.89 (m, 6H), 10.93 (s, 1H); MS: m/z $382\left(\mathrm{M}^{+}\right)$; Anal. Calcd.: C, 40.63; H, 2.62; N, 10.93; found: C, 40.66; H, 2.65; N, $10.96 \%$.

$3 e$

IR $\left(\mathrm{KBr}, \mathrm{cm}^{-1}: 3164,1629,1592 ;{ }^{1} \mathrm{H}\right.$ NMR (DMSO): $\delta 2.3$ (s, 3H), $3.52(\mathrm{~s}, 3 \mathrm{H}), 6.91-7.86(\mathrm{~m}$, 4H), 10.9 (s, 1H); MS: m/z $412\left(\mathrm{M}^{+}\right)$; Anal. Calcd.: C, 40.58; H, 2.92; N, 10.14; found: C, 40.61; H, 2.95; N, $10.17 \%$.

\section{5-(4-Bromo-5-(methylthio)thiophen-2-yl)-4- phenyl-4H-1,2,4-triazole-3-thiol 4}

A mixture of thiosemicarbazide 2 and 10 $\mathrm{mL}$ of $1 \mathrm{~N} \mathrm{NaOH}$ was heated under mild reflux for $1.5 \mathrm{hr}$. The progress of reaction was monitored by TLC. After completion of reaction the contents were cooled and poured into crushed ice. Then it was acidified with glacial acetic acid. The product was separated by filtration and crystallized from the mixture (1:1) of DMF and water to get corresponding triazole 4.

$4 a$

IR $\left(\mathrm{KBr}, \mathrm{cm}^{-1}\right): 3070,2997,1583,1517 ;{ }^{1} \mathrm{H}$ NMR (DMSO): $\delta 2.0$ (s, 3H), 3.5 (s, 3H), 6.4-7.5 (m, $5 \mathrm{H}), 14.11$ (s, $1 \mathrm{H})$; MS: m/z $412\left(\mathrm{M}^{+}\right)$; Anal. Calcd.: C, 40.58; H, 2.92; N, 10.14; found: C, 40.61; H, 2.95; N, $10.17 \%$.
$4 b$

IR $\left(\mathrm{KBr}, \mathrm{cm}^{-1}\right): 3077,2990,1578,1514 ;{ }^{1} \mathrm{H}$ NMR (DMSO): $\delta 2.01$ (s, 3H), 6.49-7.56 (m, 5H), 14.12 (s, 1H); MS: m/z $400\left(\mathrm{M}^{+}\right)$; Anal. Calcd.: C, 38.81; H, 2.25; N, 10.44; found: C, 38.84; H, 2.28; N, $10.47 \%$.

4c

IR $\left(\mathrm{KBr}, \mathrm{cm}^{-1}\right): 3081,2983,1588,1521 ;{ }^{1} \mathrm{H}$ NMR (DMSO): $\delta 2.01$ (s, 3H), 6.48-7.58 (m, 5H), 14.11 (s, 1H); MS: m/z $416\left(\mathrm{M}^{+}\right)$; Anal. Calcd.: C, 37.28; H, 2.17; N, 10.03; found: C, 37.32; H, 2.20; N, $10.07 ; \%$.

4d

IR $\left(\mathrm{KBr}, \mathrm{cm}^{-1}\right): 3085,2990,1580,1517 ;{ }^{1} \mathrm{H}$ NMR (DMSO): $\delta 2.01$ (s, 3H), 6.4-7.57 (m, 6H), 14.1 (s, 1H); MS: m/z $382\left(\mathrm{M}^{+}\right)$; Anal. Calcd.: C, 40.63; H, 2.62; N, 10.93; found: C, 40.67; H, 2.65; N, $10.96 \%$.

$4 \mathrm{e}$

IR $\left(\mathrm{KBr}, \mathrm{cm}^{-1}\right): 3074,2991,1589,1510 ;{ }^{1} \mathrm{H}$ NMR (DMSO): $\delta 2.02$ (s, 3H), 3.55 (s, 3H), 6.467.59 (m, 5H), 14.10 (s, 1H); MS: m/z $412\left(\mathrm{M}^{+}\right)$; Anal. Calcd.: C, 40.58; H, 2.92; N, 10.14; found: C, 40.62; H, 2.95; N, 10.18;\%.

(E)-3-(1-(4-Fluorophenyl)-3-(thiophen-2-yl)-1Hpyrazol-4-yl)-1-(2-hydroxyphenyl)prop-2-en-1one 10.

Equimolar amount of compound 8 (0.02 mole) and substituted o-hydroxy acetophenone ( 0.02 mole) were dissolved in $25 \mathrm{~mL}$ of alcohol in conical flask. To this reaction mixture $40 \% \mathrm{KOH}$ $(10 \mathrm{~mL})$ was added. The reaction mixture was stirred at room temperature for $48 \mathrm{hrs}$. The contents were then poured into crushed ice and neutralized with acetic acid. The yellow solid thus obtained was filtered and crystallized from alcohol to afford compound 10.

$10 a$

IR $\left(\mathrm{KBr}, \mathrm{cm}^{-1}\right): 3134(\mathrm{O}-\mathrm{H}), 1637(\mathrm{C}=\mathrm{O})$, 1568 (C=N), 1556 (-C=C-), 1508 (-C=C-, aromatic), 1155 (Ar-F); ${ }^{1} \mathrm{H}$ NMR (DMSO- $\left.d_{6}\right)$ : d 7.26- 8.22 (m, $11 \mathrm{H}, \mathrm{Ar}-\mathrm{H}$ and $=\mathrm{CH}), 9.44(\mathrm{~s}, 1 \mathrm{H}), 13.2$ (s, 1H, -OH); MS: m/z $458\left(\mathrm{M}^{+}\right)$;; Anal. Calcd.: C, 57.53; H, 2.85; N, 6.10; found: C, 57.56; H, 2.88; N, $6.13 \%$. 
$10 b$

IR $\left(\mathrm{KBr}, \mathrm{cm}^{-1}\right): 3132(\mathrm{O}-\mathrm{H}), 1633(\mathrm{C}=\mathrm{O})$, $1566(\mathrm{C}=\mathrm{N}), 1549$ (-C=C-), 1511 (-C=C-, aromatic), 1158 (Ar-F); ${ }^{1} \mathrm{H}$ NMR (DMSO- $d_{6}$ ): d 7.25- 8.34 (m, $12 \mathrm{H}, \mathrm{Ar}-\mathrm{H}$ and $=\mathrm{CH}), 9.47(\mathrm{~s}, 1 \mathrm{H}), 13.21(\mathrm{~s}, 1 \mathrm{H},-\mathrm{O}-$ H); MS: m/z $424\left(\mathrm{M}^{+}\right)$; Anal. Calcd.: C, 62.19; H, 3.32; $\mathrm{N}, 6.59$; found: $\mathrm{C}, 62.21 ; \mathrm{H}, 3.35 ; \mathrm{N}, 6.62 \%$.

$10 \mathrm{c}$

IR $\left(\mathrm{KBr}, \mathrm{cm}^{-1}\right)$ : $3129(\mathrm{O}-\mathrm{H}), 1631(\mathrm{C}=\mathrm{O})$, $1569(\mathrm{C}=\mathrm{N}), 1556(-\mathrm{C}=\mathrm{C}-), 1510(-\mathrm{C}=\mathrm{C}-$, aromatic), 1157 (Ar-F); ${ }^{1} \mathrm{H}$ NMR (DMSO- $\left.d_{6}\right)$ : d 7.27- 8.24 (m, $12 \mathrm{H}, \mathrm{Ar}-\mathrm{H}$ and $=\mathrm{CH}$ ), 9.45 (s, 1H), 13.22 (s, 1H, -OH); MS: m/z $468\left(\mathrm{M}^{+}\right)$; Anal. Calcd.: C, 56.30; H, 3.01; N, 5.97; found: C, 56.34; H, 3.05; N, $6.01 \%$.

10d

IR (KBr, cm $\left.{ }^{-1}\right): 3130(\mathrm{O}-\mathrm{H}), 1637(\mathrm{C}=\mathrm{O})$, $1571(\mathrm{C}=\mathrm{N}), 1551$ (-C=C-), 1501 (-C=C-, aromatic), 1149 (Ar-F); ${ }^{1} \mathrm{H}$ NMR (DMSO- $d_{6}$ ): d 2.2 (s, 3H), 7.25$8.22(\mathrm{~m}, 11 \mathrm{H}, \mathrm{Ar}-\mathrm{H}$ and $=\mathrm{CH}), 9.46(\mathrm{~s}, 1 \mathrm{H}), 13.20(\mathrm{~s}$, $1 \mathrm{H},-\mathrm{O}-\mathrm{H})$; MS: $\mathrm{m} / \mathrm{z} 438\left(\mathrm{M}^{+}\right)$; Anal. Calcd.: C, 62.94; $\mathrm{H}, 3.67$; N, 6.38; found: C, 62.98; H, 3.70; N, $6.41 \%$.

$10 \mathrm{e}$

IR $\left(\mathrm{KBr}, \mathrm{cm}^{-1}\right): 3134(\mathrm{O}-\mathrm{H}), 1641(\mathrm{C}=\mathrm{O})$, $1561(\mathrm{C}=\mathrm{N}), 1555(-\mathrm{C}=\mathrm{C}-), 1509$ (-C=C-, aromatic), 1155 (Ar-F); ${ }^{1} \mathrm{H}$ NMR (DMSO- $d_{6}$ ): d $2.24(\mathrm{~s}, 3 \mathrm{H})$, $2.29(\mathrm{~s}, 3 \mathrm{H}), 7.27-8.23(\mathrm{~m}, 11 \mathrm{H}, \mathrm{Ar}-\mathrm{H}$ and $=\mathrm{CH})$, 9.44 (s, 1H), 13.2 (s, 1H, -O-H); MS: m/z $418\left(\mathrm{M}^{+}\right)$; Anal. Calcd.: C, 68.88; H, 4.58; N, 6.69; found: C, 68.92; $\mathrm{H}, 4.62 ; \mathrm{N}, 6.73 \%$.

$10 f$

IR $\left(\mathrm{KBr}, \mathrm{cm}^{-1}\right): 3140(\mathrm{O}-\mathrm{H}), 1635(\mathrm{C}=\mathrm{O})$, 1567 (C=N), 1547 (-C=C-), 1499 (-C=C-, aromatic), 1146 (Ar-F); ${ }^{1} \mathrm{H}$ NMR (DMSO- $\left.d_{6}\right): \mathrm{d} 2.24$ (s, 3H), 7.21- $8.26(\mathrm{~m}, 12 \mathrm{H}, \mathrm{Ar}-\mathrm{H}$ and $=\mathrm{CH}), 9.45(\mathrm{~s}, 1 \mathrm{H})$, 13.20 (s, 1H, -O-H); MS: m/z $404\left(\mathrm{M}^{+}\right)$; Anal. Calcd.: C, 68.30; H, 4.24; N, 6.93; found: C, 68.34; H, 4.28; $\mathrm{N}, 6.97 \%$.

2-(1-(4-Fluorophenyl)-3-(thiophen-2-yl)-1H-
pyrazol-4-yl)-4H-chromen-4-one 11
Compound 10 (0.001 mole) was dissolved in $15 \mathrm{ml} \mathrm{DMSO}$. To this reaction mixture catalytic amount of iodine $(0.01 \mathrm{gm})$ was added. The reaction mixture was heated to 100 to $110^{\circ} \mathrm{C}$ for $1.5 \mathrm{hrs}$ and left overnight. Then $100 \mathrm{ml}$ ice cold water was added in it. The solid thus obtained was filtered and washed with dil. sodium thiosulphate followed by water. The product was crystallized from alcohol to afford compounds 11.

$11 a$

IR $\left(\mathrm{KBr}, \mathrm{cm}^{-1}\right): 1655(\mathrm{C}=\mathrm{O}), 1567(\mathrm{C}=\mathrm{N})$, $1513(\mathrm{C}=\mathrm{C}), 1158$ (Ar-F), ${ }^{1} \mathrm{H}$ NMR (DMSO- $d_{6}$ ): $\delta$ $6.77(\mathrm{~s}, 1 \mathrm{H}, \mathrm{Ar}-\mathrm{H}), 7.14-8.16(\mathrm{~m}, 9 \mathrm{H}, \mathrm{Ar}-\mathrm{H}), 9.22(\mathrm{~s}$, $1 \mathrm{H})$; MS: $\mathrm{m} / \mathrm{z} 456\left(\mathrm{M}^{+}\right)$; Anal. Calcd.: $\mathrm{C}, 57.78 ; \mathrm{H}$, 2.42; N, 6.13; found: C, 57.82; H, 2.46; N, $6.17 \%$.

\section{1b}

IR $\left(\mathrm{KBr}, \mathrm{cm}^{-1}\right)$ : $1649(\mathrm{C}=\mathrm{O}), 1561(\mathrm{C}=\mathrm{N}), 1508(\mathrm{C}=\mathrm{C})$, 1156 (Ar-F), ${ }^{1} \mathrm{H}$ NMR (DMSO- $\left.d_{6}\right): \delta 6.71(\mathrm{~s}, 1 \mathrm{H}, \mathrm{Ar}-$ H), 7.13-8.18 (m, 10H, Ar-H), 9.24 (s, 1H); MS: m/z $422\left(\mathrm{M}^{+}\right)$; Anal. Calcd.: C, 62.49; H, 2.86; N, 6.62; found: C, $62.53 ; \mathrm{H}, 2.89 ; \mathrm{N}, 6.65 \%$.

$11 \mathrm{c}$

IR $\left(\mathrm{KBr}, \mathrm{cm}^{-1}\right): 1651(\mathrm{C}=\mathrm{O}), 1562(\mathrm{C}=\mathrm{N})$, $1516(\mathrm{C}=\mathrm{C}), 1149$ (Ar-F), ${ }^{1} \mathrm{H}$ NMR (DMSO- $\left.d_{6}\right): \delta$ 6.73 (s, 1H, Ar-H), 7.15-8.13 (m, 10H, Ar-H), 9.23 (s, 1H); MS: m/z $466\left(\mathrm{M}^{+}\right)$; Anal. Calcd.: C, 56.54; H, 2.59; N, 5.99; found: C, 56.57; H, 2.62; N, $6.02 \%$.

\section{1d}

IR $\left(\mathrm{KBr}, \mathrm{cm}^{-1}\right): 1659(\mathrm{C}=\mathrm{O}), 1571(\mathrm{C}=\mathrm{N})$, $1510(\mathrm{C}=\mathrm{C}), 1148$ (Ar-F), ${ }^{1} \mathrm{H}$ NMR (DMSO- $\left.d_{6}\right): \delta$ $6.77(\mathrm{~s}, 1 \mathrm{H}, \mathrm{Ar}-\mathrm{H}), 7.15-8.19(\mathrm{~m}, 9 \mathrm{H}, \mathrm{Ar}-\mathrm{H}), 9.23(\mathrm{~s}$, $1 \mathrm{H})$; $\mathrm{MS}$ : $\mathrm{m} / \mathrm{z} 436\left(\mathrm{M}^{+}\right)$; Anal. Calcd.: $\mathrm{C}, 63.23 ; \mathrm{H}$, 3.23; N, 6.41; found: C, 63.27; H, 3.27; N, 6.45\%.

$11 \mathrm{e}$

IR $\left(\mathrm{KBr}, \mathrm{cm}^{-1}\right): 1645(\mathrm{C}=\mathrm{O}), 1569(\mathrm{C}=\mathrm{N})$, $1513(\mathrm{C}=\mathrm{C}), 1155$ (Ar-F), ${ }^{1} \mathrm{H}$ NMR (DMSO- $d_{6}$ ): $\delta$ $2.23(\mathrm{~s}, 3 \mathrm{H}), 2.29(\mathrm{~s}, 3 \mathrm{H}), 6.79(\mathrm{~s}, 1 \mathrm{H}, \mathrm{Ar}-\mathrm{H}), 7.13-$ $8.15(\mathrm{~m}, 9 \mathrm{H}, \mathrm{Ar}-\mathrm{H}), 9.21(\mathrm{~s}, 1 \mathrm{H})$; MS: m/z $416\left(\mathrm{M}^{+}\right)$; Anal. Calcd.: C, 69.21; H, 4.11; N, 6.73; found: C, $69.25 ; \mathrm{H}, 4.14 ; \mathrm{N}, 6.76 \%$.

\section{$11 f$}

IR $\left(\mathrm{KBr}, \mathrm{cm}^{-1}\right): 1650(\mathrm{C}=\mathrm{O}), 1562(\mathrm{C}=\mathrm{N})$, $1507(\mathrm{C}=\mathrm{C}), 1146(\mathrm{Ar}-\mathrm{F}),{ }^{1} \mathrm{H}$ NMR (DMSO- $\left.d_{6}\right): \delta$ $2.23(\mathrm{~s}, 3 \mathrm{H}), 6.78(\mathrm{~s}, 1 \mathrm{H}, \mathrm{Ar}-\mathrm{H}), 7.14-8.17(\mathrm{~m}, 10 \mathrm{H}$, Ar-H), 9.23 (s, 1H); MS: m/z $402\left(\mathrm{M}^{+}\right)$; Anal. Calcd.: C, 68.64; H, 3.76; N, 6.96; found: C, 68.68; H, 3.79; $\mathrm{N}, 6.99 \%$. 
Table 1: Characterization data of synthesized compounds

\begin{tabular}{|c|c|c|c|c|c|}
\hline Compd & $\mathrm{R}_{1}$ & $\mathbf{R}_{2}$ & $\mathbf{R}_{3}$ & m.p. $\left({ }^{\circ} \mathrm{C}\right)$ & Yield (\%) \\
\hline $2 a$ & $\mathrm{H}$ & $\mathrm{OCH}_{3}$ & $\mathrm{H}$ & 186 & 65 \\
\hline $2 b$ & $\mathrm{H}$ & $\mathrm{H}^{3}$ & $\mathrm{~F}$ & 180 & 74 \\
\hline $2 c$ & $\mathrm{H}$ & $\mathrm{H}$ & $\mathrm{Cl}$ & 184 & 71 \\
\hline $2 d$ & $\mathrm{H}$ & $\mathrm{H}$ & $\mathrm{H}$ & 179 & 73 \\
\hline $2 e$ & $\mathrm{OCH}_{3}$ & $\mathrm{H}$ & $\mathrm{H}$ & 155 & 61 \\
\hline $3 a$ & $\mathrm{H}$ & $\mathrm{OCH}_{3}$ & $\mathrm{H}$ & 180 & 60 \\
\hline $3 b$ & $\mathrm{H}$ & $\mathrm{H}$ & $\mathrm{F}$ & 199 & 65 \\
\hline $3 c$ & $\mathrm{H}$ & $\mathrm{H}$ & $\mathrm{Cl}$ & 172 & 73 \\
\hline $3 d$ & $\mathrm{H}$ & $\mathrm{H}$ & $\mathrm{H}$ & 177 & 60 \\
\hline $3 e$ & $\mathrm{OCH}_{3}$ & $\mathrm{H}$ & $\mathrm{H}$ & 190 & 62 \\
\hline $4 a$ & $\mathrm{H}^{3}$ & $\mathrm{OCH}_{3}$ & $\mathrm{H}$ & 235 & 68 \\
\hline $4 b$ & $\mathrm{H}$ & $\mathrm{H}^{3}$ & $\mathrm{~F}$ & 209 & 72 \\
\hline $4 c$ & $\mathrm{H}$ & $\mathrm{H}$ & $\mathrm{Cl}$ & 222 & 56 \\
\hline $4 d$ & $\mathrm{H}$ & $\mathrm{H}$ & $\mathrm{H}$ & 240 & 60 \\
\hline $4 e$ & $\mathrm{OCH}_{3}$ & $\mathrm{H}$ & $\mathrm{H}$ & 200 & 66 \\
\hline $10 a$ & $\mathrm{Cl}^{3}$ & $\mathrm{H}$ & $\mathrm{Cl}$ & 218 & 72 \\
\hline $10 b$ & $\mathrm{H}$ & $\mathrm{H}$ & $\mathrm{Cl}$ & 186 & 69 \\
\hline $10 c$ & $\mathrm{H}$ & $\mathrm{H}$ & $\mathrm{Br}$ & 178 & 66 \\
\hline $10 d$ & $\mathrm{H}$ & $\mathrm{CH}_{3}$ & $\mathrm{Cl}$ & 158 & 69 \\
\hline $10 e$ & $\mathrm{CH}_{3}$ & $\mathrm{H}^{\circ}$ & $\mathrm{CH}_{3}$ & 160 & 68 \\
\hline $10 f$ & $\mathrm{H}^{3}$ & $\mathrm{CH}_{3}$ & $\mathrm{H}^{3}$ & 160 & 63 \\
\hline $11 a$ & $\mathrm{Cl}$ & $\mathrm{H}^{3}$ & $\mathrm{Cl}$ & 258 & 62 \\
\hline $11 b$ & $\mathrm{H}$ & $\mathrm{H}$ & $\mathrm{Cl}$ & 264 & 68 \\
\hline $11 \mathrm{c}$ & $\mathrm{H}$ & $\mathrm{H}$ & $\mathrm{Br}$ & 254 & 67 \\
\hline $11 d$ & $\mathrm{H}$ & $\mathrm{CH}_{3}$ & $\mathrm{Cl}$ & 258 & 69 \\
\hline $11 \mathrm{e}$ & $\mathrm{CH}_{3}$ & $\mathrm{H}^{3}$ & $\mathrm{CH}_{3}$ & 238 & 65 \\
\hline $11 f$ & $\mathrm{H}^{\circ}$ & $\mathrm{CH}_{3}$ & $\mathrm{H}^{\circ}$ & 196 & 61 \\
\hline $12 a$ & $\mathrm{Cl}$ & $\mathrm{H}^{3}$ & $\mathrm{Cl}$ & 190 & 67 \\
\hline $12 b$ & $\mathrm{H}$ & $\mathrm{H}$ & $\mathrm{Cl}$ & 171 & 63 \\
\hline $12 c$ & $\mathrm{H}$ & $\mathrm{H}$ & $\mathrm{Br}$ & 194 & 70 \\
\hline $12 d$ & $\mathrm{H}$ & $\mathrm{CH}_{3}$ & $\mathrm{Cl}$ & 178 & 69 \\
\hline $12 e$ & $\mathrm{CH}_{3}$ & $\mathrm{H}^{3}$ & $\mathrm{CH}_{3}$ & 156 & 62 \\
\hline $12 f$ & $\mathrm{H}^{3}$ & $\mathrm{CH} 3$ & $\mathrm{H}^{3}$ & 130 & 59 \\
\hline $13 a$ & $\mathrm{Cl}$ & $\mathrm{H}$ & $\mathrm{Cl}$ & 230 & 73 \\
\hline $13 b$ & $\mathrm{H}$ & $\mathrm{H}$ & $\mathrm{Cl}$ & 188 & 78 \\
\hline $13 c$ & $\mathrm{H}$ & $\mathrm{H}$ & $\mathrm{Br}$ & 200 & 74 \\
\hline $13 d$ & $\mathrm{H}$ & $\mathrm{CH}_{3}$ & $\mathrm{Cl}$ & 208 & 71 \\
\hline $13 e$ & $\mathrm{CH}_{3}$ & $\mathrm{H}^{3}$ & $\mathrm{CH}_{3}$ & 232 & 69 \\
\hline $13 f$ & $\mathrm{H}^{3}$ & $\mathrm{CH}_{3}$ & $\mathrm{H}^{3}$ & 164 & 64 \\
\hline $15 a$ & $\mathrm{Cl}$ & $\mathrm{H}^{3}$ & $\mathrm{Cl}$ & 178 & 62 \\
\hline $15 b$ & $\mathrm{H}$ & $\mathrm{H}$ & $\mathrm{Cl}$ & 154 & 67 \\
\hline $15 c$ & $\mathrm{H}$ & $\mathrm{H}$ & $\mathrm{Br}$ & 168 & 66 \\
\hline $15 d$ & $\mathrm{H}$ & $\mathrm{CH}_{3}$ & $\mathrm{Cl}$ & 160 & 61 \\
\hline $15 \mathrm{e}$ & $\mathrm{H}$ & $\mathrm{H}^{3}$ & $\mathrm{CH}_{3}$ & 142 & 58 \\
\hline $16 a$ & $\mathrm{Cl}$ & $\mathrm{H}$ & $\mathrm{Cl}^{3}$ & 250 & 69 \\
\hline $16 b$ & $\mathrm{H}$ & $\mathrm{H}$ & $\mathrm{Cl}$ & 270 & 63 \\
\hline $16 c$ & $\mathrm{H}$ & $\mathrm{H}$ & $\mathrm{Br}$ & 274 & 68 \\
\hline $16 d$ & $\mathrm{H}$ & $\mathrm{CH}_{3}$ & $\mathrm{Cl}$ & 234 & 60 \\
\hline $16 e$ & $\mathrm{H}$ & $\mathrm{H}^{\circ}$ & $\mathrm{CH}_{3}$ & 165 & 52 \\
\hline $17 a$ & $\mathrm{Cl}$ & $\mathrm{H}$ & $\mathrm{Cl}^{3}$ & 275 & 62 \\
\hline $17 b$ & $\mathrm{H}$ & $\mathrm{H}$ & $\mathrm{Cl}$ & 295 & 65 \\
\hline $17 \mathrm{c}$ & $\mathrm{H}$ & $\mathrm{H}$ & $\mathrm{Br}$ & 284 & 60 \\
\hline $17 d$ & $\mathrm{H}$ & $\mathrm{CH}_{3}$ & $\mathrm{Cl}$ & 298 & 63 \\
\hline $17 e$ & $\mathrm{H}$ & $\mathrm{H}^{3}$ & $\mathrm{CH}_{3}$ & 272 & 61 \\
\hline
\end{tabular}


2-(5-(1-(4-Fluorophenyl)-3-(thiophen-2-yl)-1Hpyrazol-4-yl)-4,5-dihydro-1H-pyrazol-3-yl)phenol 12.

Compound 10 (0.003 mol) was taken in $100 \mathrm{~mL}$ RBF with $15 \mathrm{~mL}$ dioxane. To this reaction mixture $1 \mathrm{~mL}$ hydrazine hydrate was added and the contents were heated under refluxed for 4 hours. Then to the reaction mixture $2 \mathrm{~mL}$ gl. acetic acid was and heating was continued for further 3 hours. After complete heating contents were cooled to room temperature and poured over crushed ice. The solid thus obtained was separated by filtration and crystallized with alcohol to get compounds 12 . Products obtained were identified with help of spectral data. Their characterization data is given in the Table-1.

$12 \mathrm{a}$

IR $\left(\mathrm{KBr}, \mathrm{cm}^{-1}\right): 3405(-\mathrm{O}-\mathrm{H}), 3320(-\mathrm{N}-\mathrm{H})$, 3071 (Ar-H), 1587 (C=N); ${ }^{1} \mathrm{H}$ NMR (DMSO): $\delta 3.20$ (dd, 1H), 3.74 (dd, 1H), $5.1(\mathrm{t}, 1 \mathrm{H}), 7.17-8.13(\mathrm{~m}$, $10 \mathrm{H}$, aromatic and $\mathrm{N}-\mathrm{H}$ protons), $8.58(\mathrm{~s}, 1 \mathrm{H}$, pyrazole proton), 12.00 (s, 1H, -O-H proton); MS: m/ z $472\left(\mathrm{M}^{+}\right)$; Anal. Calcd.: C, 55.82; H, 3.19; N, 11.84; found: C, 55.86; H, 3.23; N, $11.88 \%$.

$12 b$

IR $\left(\mathrm{KBr}, \mathrm{cm}^{-1}\right): 3409(-\mathrm{O}-\mathrm{H}), 3325(-\mathrm{N}-\mathrm{H})$, 3074 (Ar-H), 1580 (C=N); ${ }^{1} \mathrm{H}$ NMR (DMSO): $\delta 3.21$ (dd, $1 \mathrm{H}), 3.74(\mathrm{dd}, 1 \mathrm{H}), 5.11$ (t, $1 \mathrm{H}), 7.16-8.14(\mathrm{~m}$, $11 \mathrm{H}$, aromatic and $\mathrm{N}-\mathrm{H}$ protons), $8.57(\mathrm{~s}, 1 \mathrm{H}$, pyrazole proton), 12.01 (s, 1H, -O-H proton); MS: m/ z $438\left(\mathrm{M}^{+}\right)$; Anal. Calcd.: C, 60.20; H, 3.67; N, 12.77; found: C, $60.24 ; \mathrm{H}, 3.71 ; \mathrm{N}, 12.80 \%$.

12c

IR $\left(\mathrm{KBr}, \mathrm{cm}^{-1}\right): 3402(-\mathrm{O}-\mathrm{H}), 3322(-\mathrm{N}-\mathrm{H})$, 3070 (Ar-H), $1581(\mathrm{C}=\mathrm{N}) ;{ }^{1} \mathrm{H}$ NMR (DMSO): $\delta 3.22$ (dd, 1H), $3.74(\mathrm{dd}, 1 \mathrm{H}), 5.12(\mathrm{t}, 1 \mathrm{H}), 7.11-8.12(\mathrm{~m}$, $11 \mathrm{H}$, aromatic and $\mathrm{N}-\mathrm{H}$ protons), $8.59(\mathrm{~s}, 1 \mathrm{H}$, pyrazole proton), 12.04 (s, 1H, -O-H proton); MS: m/ z $482\left(\mathrm{M}^{+}\right)$; Anal. Calcd.: C, 54.67; H, 3.34; N, 11.59; found: C, 54.71; H, 3.38; N, $11.63 \%$.

$12 d$

IR $\left(\mathrm{KBr}, \mathrm{cm}^{-1}\right): 3410(-\mathrm{O}-\mathrm{H}), 3320(-\mathrm{N}-\mathrm{H})$, 3072 (Ar-H), $1577(\mathrm{C}=\mathrm{N}) ;{ }^{1} \mathrm{H}$ NMR (DMSO): $\delta 2.23$ $(\mathrm{s}, 3 \mathrm{H}), 3.21(\mathrm{dd}, 1 \mathrm{H}), 3.72(\mathrm{dd}, 1 \mathrm{H}), 5.11(\mathrm{t}, 1 \mathrm{H})$, 7.16-8.16 (m, $10 \mathrm{H}$, aromatic and $\mathrm{N}-\mathrm{H}$ protons), 8.57 (s, $1 \mathrm{H}$, pyrazole proton), 12.02 (s, $1 \mathrm{H},-\mathrm{O}-\mathrm{H}$ proton);
MS: m/z $452\left(\mathrm{M}^{+}\right)$; Anal. Calcd.: C, 60.99; H, 4.01; N, 12.37; found: C, 61.03; H, 4.05; N, $12.41 \%$.

$12 \mathrm{e}$

IR $\left(\mathrm{KBr}, \mathrm{cm}^{-1}\right): 3407$ (-O-H), $3324(-\mathrm{N}-\mathrm{H})$, 3065 (Ar-H), 1579 (C=N); ${ }^{1} \mathrm{H}$ NMR (DMSO): $\delta 2.23$ (s, 3H), 2.25 (s, 3H), 3.23 (dd, 1H), 3.73 (dd, 1H), $5.12(\mathrm{t}, 1 \mathrm{H}), 7.16-8.14(\mathrm{~m}, 10 \mathrm{H}$, aromatic and $\mathrm{N}-\mathrm{H}$ protons), 8.56 (s, $1 \mathrm{H}$, pyrazole proton), 12.01 (s, $1 \mathrm{H},-\mathrm{O}-\mathrm{H}$ proton); MS: m/z $432\left(\mathrm{M}^{+}\right)$; Anal. Calcd.: $\mathrm{C}$, $66.65 ; \mathrm{H}, 4.89$; N, 12.95; found: C, 66.69; H, 4.93; N, $12.99 \%$.

\section{$12 f$}

IR $\left(\mathrm{KBr}, \mathrm{cm}^{-1}\right): 3401(-\mathrm{O}-\mathrm{H}), 3325(-\mathrm{N}-\mathrm{H})$, 3066 (Ar-H), 1591 (C=N); ${ }^{H} \mathrm{H}$ NMR (DMSO): $\delta 2.24$ (s, 3H), 3.21 (dd, 1H), 3.72 (dd, 1H), $5.13(\mathrm{t}, 1 \mathrm{H})$, 7.15-8.13 $(\mathrm{m}, 11 \mathrm{H}$, aromatic and $\mathrm{N}-\mathrm{H}$ protons), 8.55 (s, 1H, pyrazole proton), 12.00 (s, $1 \mathrm{H},-\mathrm{O}-\mathrm{H}$ proton); MS: m/z $418\left(\mathrm{M}^{+}\right)$; Anal. Calcd.: C, 66.01; H, 4.58; N, 13.39; found: C, 66.05; H, 4.62; N, $13.43 \%$.

\section{2-((E)-2-(1-(4-Fluorophenyl)-3-(thiophen-2-yl)-1H- pyrazol-4-yl)-2,3-dihydrobenzo[ $b][1,4]$ thiazepin-4- yl)phenol 13.}

Compound $10(0.001 \mathrm{~mol})$ and o-amino thiophenol $(0.001 \mathrm{~mol})$ was suspended in $10 \mathrm{ml}$ ethanol. Reaction mass was heated to reflux at $90^{\circ} \mathrm{C}$ for 4-5h. To the reaction mixture $2 \mathrm{ml}$ glacial acetic acid was added and heating continued for further 3h. Contents were cooled and poured into crushed ice. The product obtained was separated by filtration and crystallized from ethanol. Products obtained were identified with help of spectral data. Their characterization data is given in the Table-1.

\section{$13 a$}

IR (KBr): 3413 (-O-H), 3066 (Ar-H), 1590 \& $1566(\mathrm{C}=\mathrm{N}), 612(\mathrm{C}-\mathrm{S}) \mathrm{cm}^{-1} ;{ }^{1} \mathrm{H}$ NMR (DMSO): $\delta$ $3.12(\mathrm{t}, 1 \mathrm{H}), 3.76(\mathrm{dd}, 1 \mathrm{H}), 5.57$ (dd, 1H), 7.18-8.05 (m, 13H, Ar-H), 8.51 (s, 1H, pyrazole proton), 15.9 (s, 1H, -O-H proton); MS: m/z $565\left(\mathrm{M}^{+}\right)$; Anal. Calcd.: C, 59.36; H, 3.20; N, 7.42; found: C, 59.40; H, 3.23; N, $7.46 ; \%$.

$13 b$

IR (KBr): $3411(-\mathrm{O}-\mathrm{H}), 3070(\mathrm{Ar}-\mathrm{H}), 1594$ \& $1570(\mathrm{C}=\mathrm{N}), 611(\mathrm{C}-\mathrm{S}) \mathrm{cm}^{-1} ;{ }^{1} \mathrm{H}$ NMR (DMSO): $\delta$ $3.11(\mathrm{t}, 1 \mathrm{H}), 3.75$ (dd, $1 \mathrm{H}), 5.57$ (dd, 1H), 7.17-8.04 (m, 14H, Ar-H), 8.50 (s, 1H, pyrazole proton), 15.95 
Table 2: Antimicrobial activity results for concentration $1000 \mu \mathrm{g} / \mathrm{ml}$

\begin{tabular}{|c|c|c|c|c|c|c|c|}
\hline \multirow[t]{2}{*}{ Compd } & \multicolumn{3}{|c|}{ Antifungal test models } & \multicolumn{4}{|c|}{ Antibacterial test models } \\
\hline & C. albica & migatus & A. Niger & S. aureus & E. coli & S. epidermidis & P. Vulgaris \\
\hline $2 a$ & 12 & 8 & 10 & 12 & 11 & 12 & - \\
\hline $2 b$ & 10 & - & 12 & 10 & 8 & 10 & 8 \\
\hline $2 c$ & - & - & 10 & - & 10 & - & - \\
\hline $2 d$ & 10 & 8 & 8 & 12 & 6 & 12 & 8 \\
\hline $2 e$ & 10 & 6 & 8 & 11 & 10 & 10 & 8 \\
\hline $3 a$ & 8 & 6 & 8 & 10 & 11 & 8 & - \\
\hline $3 b$ & 10 & - & 6 & 12 & 10 & 8 & 12 \\
\hline $3 c$ & 6 & 8 & 6 & 8 & 12 & 6 & - \\
\hline $3 d$ & 11 & 6 & 8 & 8 & 10 & 8 & 6 \\
\hline $3 e$ & 8 & - & 6 & 11 & 8 & 6 & - \\
\hline $4 a$ & 10 & 8 & 8 & 12 & 10 & 8 & 6 \\
\hline $4 b$ & 10 & 6 & 4 & 10 & 10 & 6 & - \\
\hline $4 c$ & 12 & 6 & 6 & 11 & 10 & 8 & 4 \\
\hline $4 d$ & 10 & 8 & - & 10 & 6 & 6 & 8 \\
\hline $4 e$ & 8 & 4 & 6 & 12 & 10 & 8 & 4 \\
\hline $10 a$ & 8 & 4 & 10 & 8 & 8 & 6 & 8 \\
\hline $10 b$ & 4 & 6 & 8 & 6 & 4 & 4 & - \\
\hline $10 c$ & 8 & 6 & - & 4 & 6 & 8 & 6 \\
\hline $10 d$ & 6 & 4 & - & 10 & 8 & - & - \\
\hline $10 e$ & 10 & 6 & 4 & 6 & 8 & 9 & 8 \\
\hline $10 f$ & 4 & 6 & 4 & 8 & 6 & 6 & 4 \\
\hline $11 a$ & 8 & 6 & - & $6 \mathrm{~h}$ & $8 \mathrm{~h}$ & 6 & - \\
\hline $11 \mathrm{~b}$ & 6 & 8 & 6 & 12 & 10 & 11 & 12 \\
\hline $11 \mathrm{c}$ & 11 & 6 & 8 & 10 & 6 & 8 & - \\
\hline $11 d$ & 10 & 6 & 4 & 6 & 8 & 11 & - \\
\hline $11 \mathrm{e}$ & 8 & 4 & 6 & 8 & 6 & 6 & - \\
\hline $11 \mathrm{f}$ & 4 & 8 & 6 & 6 & 7 & 4 & - \\
\hline $12 a$ & 11 & 10 & 8 & 6 & 4 & 6 & 8 \\
\hline $12 b$ & 10 & 5 & 6 & 4 & 6 & 6 & 4 \\
\hline $12 c$ & 10 & 6 & 6 & 12 & 10 & 6 & 6 \\
\hline $12 d$ & 8 & 6 & 6 & 10 & 8 & 10 & 8 \\
\hline $12 e$ & 10 & 6 & 6 & 12 & 8 & 6 & 12 \\
\hline $12 f$ & 8 & 4 & 6 & 10 & 6 & 8 & 4 \\
\hline $13 a$ & 10 & 6 & 8 & 10 & 8 & 6 & 6 \\
\hline $13 b$ & 6 & 8 & 6 & 8 & 6 & 8 & 4 \\
\hline $13 c$ & 8 & 10 & 10 & 6 & 8 & 10 & 6 \\
\hline $13 d$ & 8 & 4 & - & 8 & 10 & 8 & - \\
\hline $13 e$ & 6 & 8 & - & 8 & 10 & 6 & - \\
\hline $13 f$ & 6 & 7 & - & 8 & 6 & 10 & 6 \\
\hline $15 a$ & 8 & 8 & 6 & 10 & 8 & 6 & - \\
\hline $15 b$ & 6 & 6 & 4 & 9 & 6 & 8 & - \\
\hline $15 c$ & 8 & 6 & - & 10 & 6 & 4 & - \\
\hline $15 d$ & 8 & 8 & 8 & 4 & 6 & 10 & - \\
\hline $15 e$ & 8 & 4 & - & 4 & 7 & 8 & - \\
\hline $16 a$ & 12 & 8 & 4 & 6 & 8 & 10 & 4 \\
\hline
\end{tabular}




\begin{tabular}{|c|c|c|c|c|c|c|c|}
\hline $16 b$ & 12 & 10 & 8 & 6 & 4 & 6 & - \\
\hline $16 \mathrm{c}$ & 8 & 6 & 6 & 12 & 10 & 13 & - \\
\hline $16 d$ & 10 & 8 & 6 & 12 & 10 & 10 & - \\
\hline $16 e$ & 10 & 8 & 6 & 10 & 8 & 6 & 6 \\
\hline $17 a$ & $10 \mathrm{~h}$ & 8 & 6 & $6 \mathrm{~h}$ & $8 \mathrm{~h}$ & 8 & 4 \\
\hline $17 b$ & 12 & $8 \mathrm{~h}$ & - & 10 & $12 \mathrm{~h}$ & 10 & 8 \\
\hline $17 c$ & 12 & 6 & - & 8 & 12 & 6 & - \\
\hline $17 d$ & 6 & 4 & 6 & 7 & 9 & 6 & - \\
\hline $17 e$ & 12 & 6 & 8 & 11 & 14 & 16 & - \\
\hline \multicolumn{8}{|l|}{ Fluconazole } \\
\hline $10 \mu \mathrm{g} / \mathrm{disc}$ & 24 & 14 & 20 & NA & NA & NA & NA \\
\hline Amikacin20 $\mu \mathrm{g} / \mathrm{ml}$ & NA & NA & NA & 28 & 20 & 23 & 18 \\
\hline
\end{tabular}

NT: Not tested, h: hazy.

(s, 1H, -O-H proton); MS: m/z $531\left(\mathrm{M}^{+}\right)$; Anal. Calcd.: C, 63.21; H, 3.60; N, 7.90; found: C, 63.25; H, 3.64; N, $7.94 \%$.

$13 c$

IR (KBr): 3409 (-O-H), $3062(\mathrm{Ar}-\mathrm{H}), 1597$ \& $1562(\mathrm{C}=\mathrm{N}), 616(\mathrm{C}-\mathrm{S}) \mathrm{cm}^{-1} ;{ }^{1} \mathrm{H}$ NMR (DMSO): $\delta$ 3.12 (t, 1H), 3.77 (dd, 1H), 5.54 (dd, 1H), 7.16-8.07 (m, 14H, Ar-H), 8.52 (s, 1H, pyrazole proton), 15.93 (s, 1H, -O-H proton); MS: m/z $575\left(\mathrm{M}^{+}\right)$; Anal. Calcd.: C, 58.33; H, 3.32; N, 7.29; found: C, 58.37; H, 3.36; N, $7.33 \%$.

$13 d$

IR (KBr): 3407 (-O-H), 3068 (Ar-H), 1590 \& $1568(\mathrm{C}=\mathrm{N}), 611(\mathrm{C}-\mathrm{S}) \mathrm{cm}^{-1} ;{ }^{1} \mathrm{H}$ NMR (DMSO): $\delta$ 2.24 (s, 3H), 3.10 (t, 1H), 3.73 (dd, 1H), 5.57 (dd, $1 \mathrm{H}), 7.17-8.07$ (m, 13H, Ar-H), 8.50 (s, 1H, pyrazole proton), 15.92 (s, 1H, -O-H proton); MS: m/z 545 $\left(\mathrm{M}^{+}\right)$; Anal. Calcd.: C, 63.78; H, 3.88; N, 7.69; found: C, 63.82; H, 3.92; N, $7.73 \%$.
$13 e$

IR (KBr): $3410(-\mathrm{O}-\mathrm{H}), 3066(\mathrm{Ar}-\mathrm{H}), 1594$ \& $1565(\mathrm{C}=\mathrm{N}), 610(\mathrm{C}-\mathrm{S}) \mathrm{cm}^{-1} ;{ }^{1} \mathrm{H}$ NMR (DMSO): $\delta$ $2.24(\mathrm{~s}, 3 \mathrm{H}), 2.26(\mathrm{~s}, 3 \mathrm{H}), 3.13(\mathrm{t}, 1 \mathrm{H}), 3.74(\mathrm{dd}, 1 \mathrm{H})$, 5.56 (dd, 1H), 7.16-8.01 (m, 13H, Ar-H), 8.53 (s, 1H, pyrazole proton), 15.91 (s, $1 \mathrm{H},-\mathrm{O}-\mathrm{H}$ proton); MS: $\mathrm{m} /$ z $525\left(\mathrm{M}^{+}\right)$; Anal. Calcd.: C, 68.55; H, 4.60; N, 7.99; found: C, 68.59; H, 4.64; N, $8.03 \%$.

$13 f$

IR (KBr): 3404 (-O-H), $3062(\mathrm{Ar}-\mathrm{H}), 1584$ \& $1559(\mathrm{C}=\mathrm{N}), 610(\mathrm{C}-\mathrm{S}) \mathrm{cm}^{-1} ;{ }^{1} \mathrm{H}$ NMR $(\mathrm{DMSO}): \delta$ $2.21(\mathrm{~s}, 3 \mathrm{H}), 3.12(\mathrm{t}, 1 \mathrm{H}), 3.74$ (dd, $1 \mathrm{H}), 5.57$ (dd, 1H), 7.14-8.03 (m, 14H, Ar-H), 8.54 (s, 1H, pyrazole proton), 15.90 (s, 1H, -O-H proton); MS: m/z 511 $\left(\mathrm{M}^{+}\right)$; Anal. Calcd.: C, 68.08; H, 4.33; N, 8.21; found: C, $68.11 ; \mathrm{H}, 4.37 ; \mathrm{N}, 8.24 \%$.

(E)-2-Acetylphenyl 3-(1-(4-fluorophenyl)-3(thiophen-2-yl)-1H-pyrazol-4-yl)acrylate 15

Equimolar amount (0.05 moles) of the compounds 14 and substituted 2-hydroxy

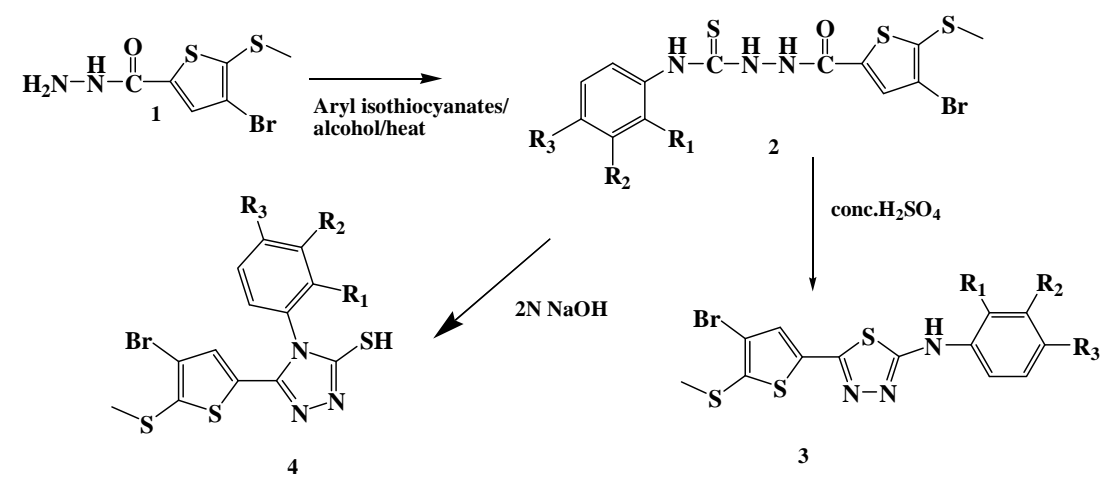

Scheme 1: 
acetophenone were taken in dry beaker. To this mixture was dissolved in $15 \mathrm{ml}$ dry pyridine. The reaction mixture was then cooled to $0^{\circ} \mathrm{C}$. To this reaction mixture $\mathrm{POCl}_{3}$ (0.06 moles) was added drop wise maintaining temperature below $10^{\circ} \mathrm{C}$. Then reaction mixture was kept overnight at room temperature. It was then poured over crushed ice with vigorous stirring. Product was separated by filtration, washed with ice-cold water and then with $2 \%$ ice-cold solution of $\mathrm{NaOH}$ followed by ice-cold water again. Purification by crystallization after drying with alcohol afforded 15.

\section{$15 a$}

IR $\left(\mathrm{KBr}, \mathrm{cm}^{-1}\right): 3120(\mathrm{O}-\mathrm{H}), 1736(\mathrm{C}=\mathrm{O})$, 1549 (C=N), 1545 (-C=C-), 1499 (-C=C-, aromatic), 1147 (Ar-F); ${ }^{1} \mathrm{H}$ NMR (DMSO- $\left.d_{6}\right): \mathrm{d} 2.21$ (s, 3H), 7.05- $8.30(\mathrm{~m}, 11 \mathrm{H}, \mathrm{Ar}-\mathrm{H}$ and $=\mathrm{CH}), 8.44(\mathrm{~s}, 1 \mathrm{H}$, pyrazole proton); MS: m/z $500\left(\mathrm{M}^{+}\right)$; Anal. Calcd.: C, 57.50; H, 3.02; N, 5.59; found: C, 57.54; H, 3.06; N, $5.63 \%$.

$15 b$

IR $\left(\mathrm{KBr}, \mathrm{cm}^{-1}\right): 3129(\mathrm{O}-\mathrm{H}), 1734(\mathrm{C}=\mathrm{O})$, $1554(\mathrm{C}=\mathrm{N}), 1544$ (-C=C-), 1494 (-C=C-, aromatic), 1143 (Ar-F); ${ }^{1} \mathrm{H}$ NMR (DMSO- $d_{6}$ ): d 2.20 (s, 3H),
7.08- $8.35(\mathrm{~m}, 12 \mathrm{H}, \mathrm{Ar}-\mathrm{H}$ and $=\mathrm{CH}), 8.48(\mathrm{~s}, 1 \mathrm{H}$, pyrazole proton); MS: m/z $466\left(\mathrm{M}^{+}\right)$; Anal. Calcd.: C, 61.74; H, 3.45; N, 6.00; found: C, 61.78; H, 3.48; N, $6.03 \%$.

\section{$15 c$}

IR $\left(\mathrm{KBr}, \mathrm{cm}^{-1}\right): 3124(\mathrm{O}-\mathrm{H}), 1739(\mathrm{C}=\mathrm{O})$, 1558 (C=N), 1558 (-C=C-), 1506 (-C=C-, aromatic), 1142 (Ar-F); ${ }^{1} \mathrm{H}$ NMR (DMSO- $d_{6}$ ): d 2.21 (s, 3H), 7.03- $8.31(\mathrm{~m}, 12 \mathrm{H}, \mathrm{Ar}-\mathrm{H}$ and $=\mathrm{CH}), 8.47(\mathrm{~s}, 1 \mathrm{H}$, pyrazole proton); MS: m/z $510\left(\mathrm{M}^{+}\right)$; Anal. Calcd.: C, 56.37; H, 3.15; N, 5.48; found: C, 56.40; H, 3.18; N, $5.52 \%$.

$15 d$

IR $\left(\mathrm{KBr}, \mathrm{cm}^{-1}\right): 3130(\mathrm{O}-\mathrm{H}), 1732(\mathrm{C}=\mathrm{O})$, 1551 (C=N), 1549 (-C=C-), 1495 (-C=C-, aromatic), 1141 (Ar-F); ${ }^{1} \mathrm{H}$ NMR (DMSO- $d_{6}$ ): d 2.20 (s, 3H), 7.03- $8.37(\mathrm{~m}, 11 \mathrm{H}, \mathrm{Ar}-\mathrm{H}$ and $=\mathrm{CH}), 8.49(\mathrm{~s}, 1 \mathrm{H}$, pyrazole proton); MS: m/z $480\left(\mathrm{M}^{+}\right)$; Anal. Calcd.: C, 62.43; H, 3.77; N, 5.82; found: C, 62.47; H, 3.80; N, $5.85 \%$.

\section{$15 e$}

IR $\left(\mathrm{KBr}, \mathrm{cm}^{-1}\right): 3135(\mathrm{O}-\mathrm{H}), 1739(\mathrm{C}=\mathrm{O})$, 1559 (C=N), 1535 (-C=C-), 1503 (-C=C-, aromatic), 1151 (Ar-F); ${ }^{1} \mathrm{H}$ NMR (DMSO- $d_{6}$ ): d 2.20 (s, 3H),

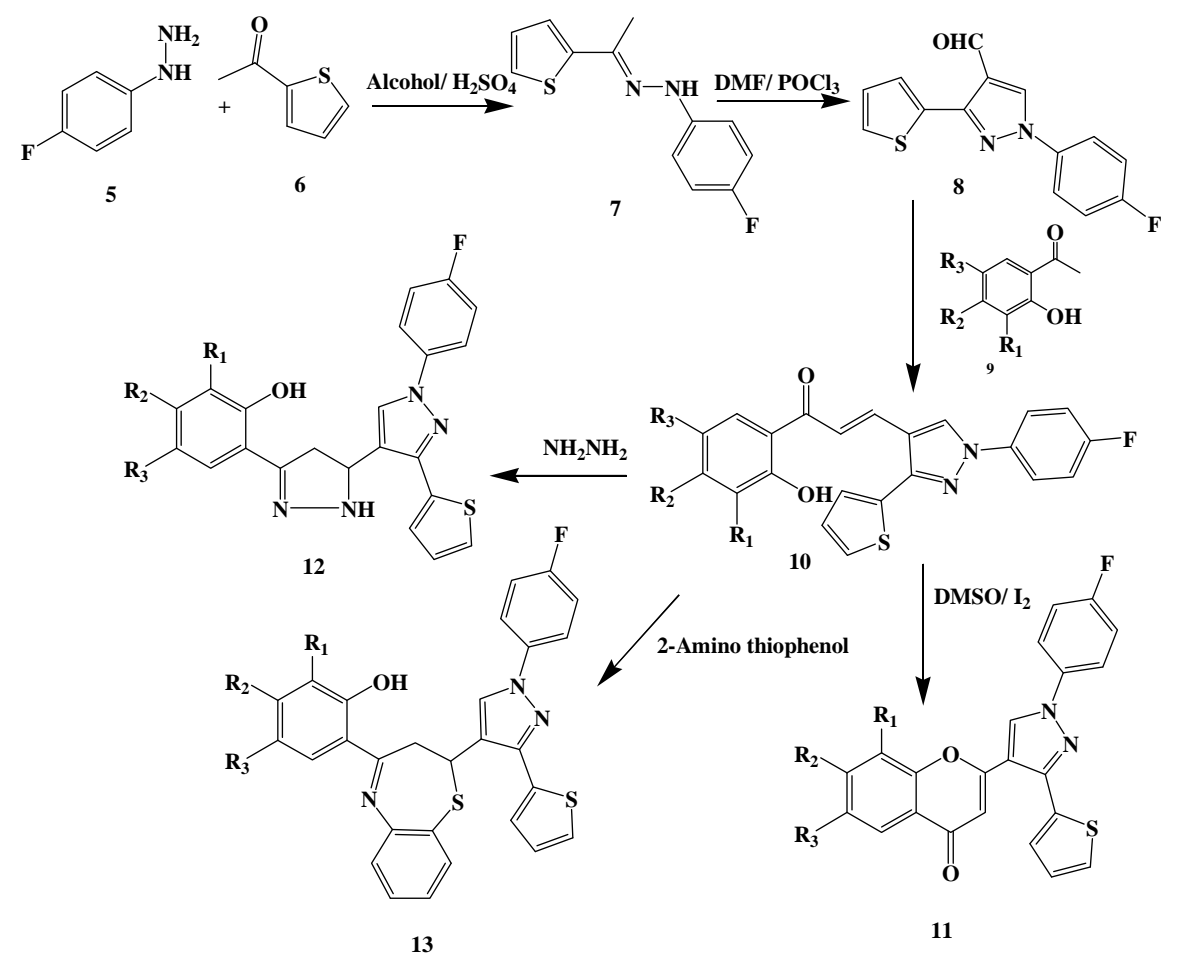

Scheme 2: 
$2.31(\mathrm{~s}, 3 \mathrm{H}), 7.00-8.27(\mathrm{~m}, 12 \mathrm{H}, \mathrm{Ar}-\mathrm{H}$ and $=\mathrm{CH})$ $8.42\left(\mathrm{~s}, 1 \mathrm{H}\right.$, pyrazole proton); MS: $\mathrm{m} / \mathrm{z} 446\left(\mathrm{M}^{+}\right)$; Anal. Calcd.: C, 67.25; H, 4.29; N, 6.27; found: C, 67.28; H, 4.33; N, $6.30 \%$.

(E)-5-(1-(4-Fluorophenyl)-3-(thiophen-2-yl)-1Hpyrazol-4-yl)-1-(2-hydroxyphenyl)pent-4-ene-1,3dione 16

Compound 15 (0.03 moles) was dissolved in $15 \mathrm{ml}$ of dry pyridine. To this mixture powdered $\mathrm{KOH}(1 \mathrm{gm})$ was added and the reaction mixture was stirred on the magnetic stirrer for 3 hours. Then it was poured over crushed ice and acidified with acetic acid. The product was then separated by filtration, washed with water, dried and crystallized with acetic acid to afford 16 .

$16 a$

IR (KBr): $3094(\mathrm{O}-\mathrm{H}), 1643(\mathrm{C}=\mathrm{O}), 1623$ ($\mathrm{C}=\mathrm{C}-), 1133(\mathrm{Ar}-\mathrm{F}) \mathrm{cm}^{-1} ;{ }^{1} \mathrm{H}$ NMR (DMSO- $\left.d_{6}\right): \delta 6.17$ $8.03(\mathrm{~m}, 11 \mathrm{H}$, Aromatic and olefinic protons), 11.54 (s, $1 \mathrm{H}$, phenolic proton), $15.21(1 \mathrm{H}$, enolic proton); MS: m/z $500\left(\mathrm{M}^{+}\right)$; Anal. Calcd.: C, 57.50; H, 3.02; N, 5.59; found: C, 57.54; H, 3.06; N, $5.63 \%$.
$16 b$

IR (KBr): $3105(\mathrm{O}-\mathrm{H}), 1649(\mathrm{C}=\mathrm{O}), 1620(-$ $\mathrm{C}=\mathrm{C}-), 1140$ (Ar-F) $\mathrm{cm}^{-1} ;{ }^{1} \mathrm{H}$ NMR (DMSO- $\left.d_{6}\right): \delta 6.11-$ $8.10(\mathrm{~m}, 12 \mathrm{H}$, Aromatic and olefinic protons), 11.58 (s, $1 \mathrm{H}$, phenolic proton), 15.20 (1H, enolic proton); MS: m/z $466\left(\mathrm{M}^{+}\right)$; Anal. Calcd.: C, 61.74; H, 3.45; N, 6.00; found: C, 61.78; H, 3.49; N, $6.04 \%$.

$16 \mathrm{c}$

IR (KBr): $3087(\mathrm{O}-\mathrm{H}), 1635(\mathrm{C}=\mathrm{O}), 1620(-$ $\mathrm{C}=\mathrm{C}-), 1123(\mathrm{Ar}-\mathrm{F}) \mathrm{cm}^{-1} ;{ }^{1} \mathrm{H}$ NMR (DMSO- $\left.d_{6}\right): \delta 6.12-$ $8.06(\mathrm{~m}, 12 \mathrm{H}$, Aromatic and olefinic protons), 11.55 (s, $1 \mathrm{H}$, phenolic proton), $15.19(1 \mathrm{H}$, enolic proton); MS: m/z $510\left(\mathrm{M}^{+}\right)$; Anal. Calcd.: C, 56.37; H, 3.15; N, 5.48; found: C, 56.40; H, 3.18; N, $5.51 \%$.

$16 d$

IR (KBr): $3101(\mathrm{O}-\mathrm{H}), 1652(\mathrm{C}=\mathrm{O}), 1626$ ($\mathrm{C}=\mathrm{C}-), 1142(\mathrm{Ar}-\mathrm{F}) \mathrm{cm}^{-1} ;{ }^{1} \mathrm{H}$ NMR (DMSO- $\left.d_{6}\right): \delta 2.31$ $(\mathrm{s}, 3 \mathrm{H}), 6.20-8.12(\mathrm{~m}, 11 \mathrm{H}$, Aromatic and olefinic protons), 11.49 (s, $1 \mathrm{H}$, phenolic proton), $15.25(1 \mathrm{H}$, enolic proton); MS: m/z $480\left(\mathrm{M}^{+}\right)$; Anal. Calcd.: C, 62.43; H, 3.77; N, 5.82; found: C, 62.47; H, 3.81; N, $5.86 \%$.

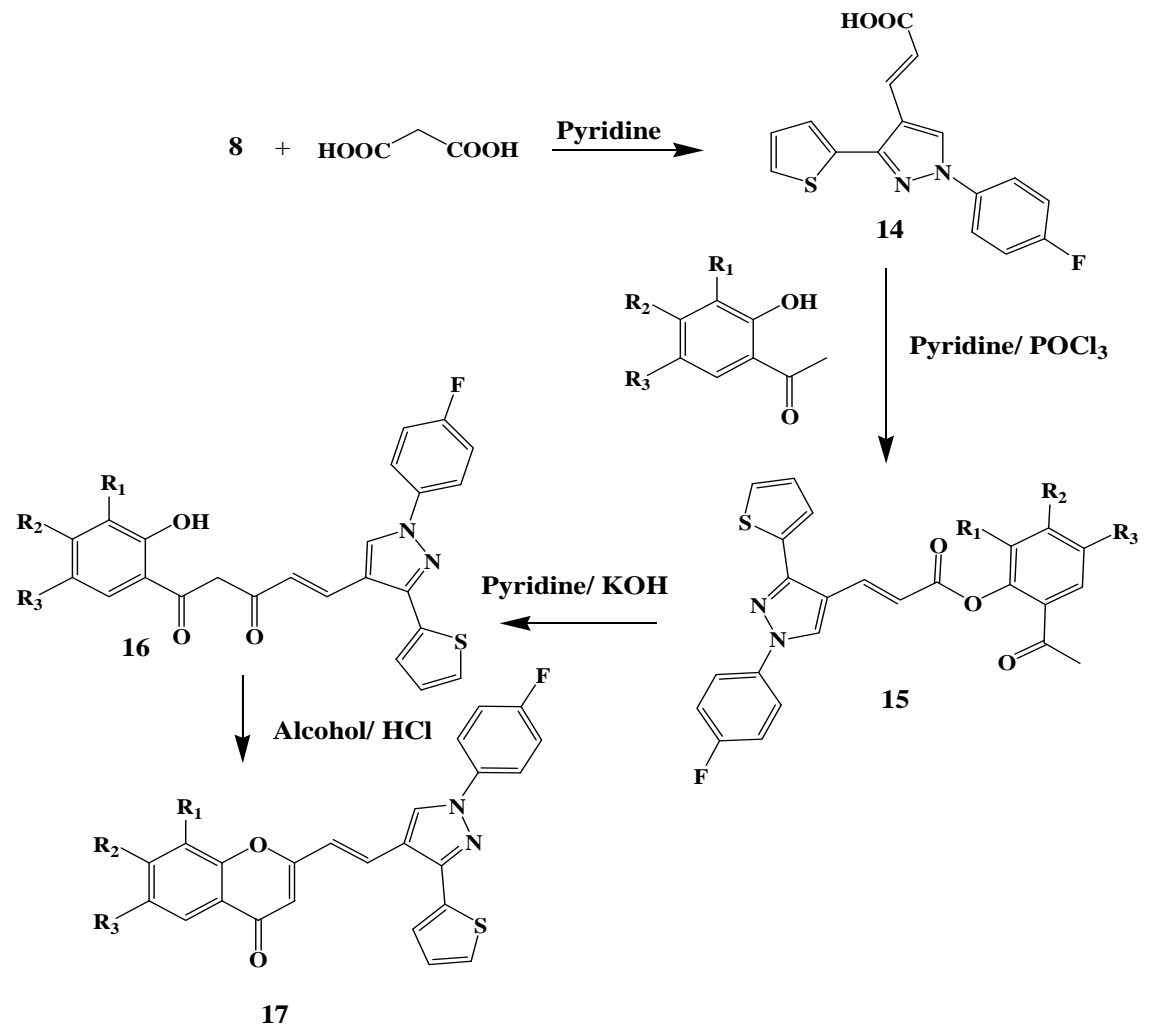

Scheme 3: 
$16 e$

IR (KBr): $3087(\mathrm{O}-\mathrm{H}), 1633(\mathrm{C}=\mathrm{O}), 1627(-$ $\mathrm{C}=\mathrm{C}-), 1147(\mathrm{Ar}-\mathrm{F}) \mathrm{cm}^{-1} ;{ }^{1} \mathrm{H}$ NMR (DMSO- $\left.d_{6}\right): \delta 2.20$ $(\mathrm{s}, 3 \mathrm{H}), 6.23-8.01(\mathrm{~m}, 12 \mathrm{H}$, Aromatic and olefinic protons), 11.48 (s, $1 \mathrm{H}$, phenolic proton), $15.21(1 \mathrm{H}$, enolic proton); MS: m/z $446\left(\mathrm{M}^{+}\right)$; Anal. Calcd.: C, 67.25; H, 4.29; N, 6.27; found: C, 67.28; H, 4.32; N, $6.30 \%$.

2-((E)-2-(1-(4-Fluorophenyl)-3-(thiophen-2-yl)-1Hpyrazol-4-yl)vinyl)-4H-chromen-4-one 17

Compound 16 (0.01 moles) was dissolved in $15 \mathrm{ml}$ glacial acetic acid in RBF. To this reaction mixture $1 \mathrm{ml}$ conc. $\mathrm{HCl}$ was added and contents were refluxed for 2 hours. Then it was cooled and poured over crushed ice. The product was then separated by filtration, washed with water, dried and crystallized with acetic acid to afford 17.

$17 a$

IR $\left(\mathrm{KBr}, \mathrm{cm}^{-1}\right): 1651(\mathrm{C}=\mathrm{O}), 1559(\mathrm{C}=\mathrm{N})$, 1505 (C=C), 1149 (Ar-F); ${ }^{1} \mathrm{H}$ NMR (DMSO- $d_{6}$ ): 6.64 (s, 1H, Ar-H), 7.01-8.52 (m, 11H, Ar-H), $9.12(\mathrm{~s}, 1 \mathrm{H})$; MS: m/z $482\left(\mathrm{M}^{+}\right)$; Anal. Calcd.: C, 59.64; H, 2.71; N, 5.80; found: C, 57.82; H, 2.46; N, $6.17 \%$.

$17 b$

IR $\left(\mathrm{KBr}, \mathrm{cm}^{-1}\right): 1654(\mathrm{C}=\mathrm{O}), 1548(\mathrm{C}=\mathrm{N})$,
1509 (C=C), 1156 (Ar-F); ${ }^{1} \mathrm{H}$ NMR (DMSO- $d_{6}$ ): d $6.65(\mathrm{~s}, 1 \mathrm{H}, \mathrm{Ar}-\mathrm{H}), 7.07-8.40(\mathrm{~m}, 12 \mathrm{H}, \mathrm{Ar}-\mathrm{H}), 9.16$ (s, 1H); MS: m/z $448\left(\mathrm{M}^{+}\right)$; Anal. Calcd.: C, 64.21; H, 3.14; N, 6.24; found: C, 64.24; H, 3.17; N, $6.27 \%$.

$17 \mathrm{c}$

IR $\left(\mathrm{KBr}, \mathrm{cm}^{-1}\right): 1659(\mathrm{C}=\mathrm{O}), 1557(\mathrm{C}=\mathrm{N})$, $1501(\mathrm{C}=\mathrm{C}), 1152(\mathrm{Ar}-\mathrm{F}) ;{ }^{1} \mathrm{H}$ NMR (DMSO- $\left.d_{6}\right): \mathrm{d}$ 6.63 (s, 1H, Ar-H), 7.05-8.50 (m, 12H, Ar-H), 9.13 (s, $1 \mathrm{H})$; MS: m/z $500\left(\mathrm{M}^{+}\right)$; Anal. Calcd.: C, 58.43; $\mathrm{H}$, 2.86; N, 5.68; found: C, 58.46; H, 2.89; N, $5.71 \%$.

$17 d$

IR $\left(\mathrm{KBr}, \mathrm{cm}^{-1}\right): 1653(\mathrm{C}=\mathrm{O}), 1549(\mathrm{C}=\mathrm{N})$, $1498(\mathrm{C}=\mathrm{C}), 1141$ (Ar-F); ${ }^{1} \mathrm{H}$ NMR (DMSO- $\left.d_{6}\right)$ : d 2.31 (s, 3H), 6.65 (s, 1H, Ar-H), 7.01-8.42 (m, 11H, Ar-H), 9.15 (s, 1H); MS: m/z $462\left(\mathrm{M}^{+}\right)$; Anal. Calcd.: C, 64.86; H, 3.48; N, 6.05; found: C, 64.90; H, 3.52; N, $6.09 \%$.

$17 \mathrm{e}$

IR $\left(\mathrm{KBr}, \mathrm{cm}^{-1}\right): 1658(\mathrm{C}=\mathrm{O}), 1560(\mathrm{C}=\mathrm{N})$, $1514(\mathrm{C}=\mathrm{C}), 1145$ (Ar-F); ${ }^{1} \mathrm{H}$ NMR (DMSO- $\left.d_{6}\right)$ : d 2.28 (s, 3H), 6.61 (s, $1 \mathrm{H}, \mathrm{Ar}-\mathrm{H}), 7.04-8.39(\mathrm{~m}, 12 \mathrm{H}$, Ar-H), 9.14 (s, 1H); MS: m/z $428\left(\mathrm{M}^{+}\right)$; Anal. Calcd.: C, 70.08; H, 4.00; N, 6.54; found: C, 70.12; H, 4.03; $\mathrm{N}, 6.58 \%$.

\section{REFERENCES}

1. Ansary, A. K.; Omar, H. A. Bull. Faculty Pharm. 2001, 39, 17.

2. Kavitha, P. N.; Vijayanthimala, P.; Saravanan, J.; Mohan, S. Res. J. Pharma., Bio. and Chem. Sci. 2010, 1(2), 124-130.

3. Jose, L.; Gonzalez, C. E.; Stephens, T.; Wenzler, R. Eur. J. Med. Chem. 2007, 42, 552-557.

4. Folkes, A. J.; Ahmadi, K.; Alderton, W. K.; Alix, S.; Baker, S. J. Journal of Medi. Chem. 2008, 51, 5522-5532.

5. Kulandasamy, R.; Adhikari, A. V.; Stables, J. P. Eur. J. Med. Chem. 2009, 44(11), 43764384.

6. Oganesyan, E. T.; Saraf, A. S.; Ivchenko, A. V. Pharma. Chem. Jour. 1993, 27(1), 52-54.

7. Bhatnagar, S.; Sahi, S.; Kackar, P.; Kaushik,
S.; Dave, M. K.; Shukla, A.; Goel, A. Bioorg. Med. Chem. Lett. 2010, 20(16), 4945-4950.

8. Pawar, S. P.; Kondhare, D. D.; Zubaidha, P.K. Med. Chem. Res. 2013, 22 (2), 753-757.

9. Gomes, A.; Fernandes, E.; Silva, A. M. S.; Pinto, D. C. G. A.; Santos, C. M. M.; Cavaleiro, J. A.S.; Lima, J. L. F. C. Biochem. Pharmaco. 2009, 78(2), 171-177.

10. Momin, M.; Ramjugernath, D.; Chenia, H.; Koorbanally, N. A. Journal of Chemistry 2013, Article ID 436758.

11. Ono, M.; Maya, Y.; Haratake, M.; Nakayama, M. Bioorg. Med. Chem. 2007, 15, 444-450.

12. Karale, B. K.; Pawar, P. Y.; Gadakh, A. V.; Akolkar, H. N.; Rindhe, S. S. Ind. Jour. Het. Chem. 2014, 23(3), 283-292.

13. Peng-Cheng, Lv.; Hai-L, Z., Huan-Qiu, Li; 
Sun, J; Zhou, Y. Bioorg. Med. Chem. 2010, 18, 4606-4614.

14. Bonesi, M; Loizzo, M. R.; Statti, G. A.; Michel, S.; Menichini, T. F. Bioorg. Med. Chem. Lett. 2010, 20, 1990-1993.

15. Zhang, K.; Wang, P.; Xuan, L. N.; Fu, X. Y.; Jing, F.; Li, S.; Liu, Y. M.; Chen, B. Q. Bioorg. Med. Chem. Lett. 2014, 24 (22), 5154-5156.

16. Jain, S. K.; Mishra, P. Eur. J. Exp. Bio. 2014, 4(2), 337-341.

17. Wu, J.; Li, J.; Xu, M. H.; Liu, D. Bioorg. Med. Chem. Lett. 2014, 24(14), 3050-3056.

18. Samel, A. B.; Pai, N. R. J. Chin. Chemi. Soc. 2010, 57, 1327-1330.

19. Gadhave, A. G.; Gaikar, R. B.; Kuchekar, S. R.; Karale, B. K. Journal of Heterocyclic Chemistry 2014, 51(6), 1849-1855.

20. Bhalgat, C. M.; Ali, M. I.; Ramesh, B.; Ramu, G. Arabian Journal of Chemistry 2014, 7, 986-993.

21. Al-Soud, Y. A.; Heydel, M.; Hartmann, R. W. Tetrahedron Letters 2011, 52(48), 6372-6375.

22. Tarzia, G.; Ocelli, E.; Toja, E.; Barone, D.; Corsico, N.; Gallico, L.; Luzzani, F. J. Med. Chem. 1988, 31(6), 1115-1123.

23. Winter, E.; Lecerf-Schmidt, F.; Gozzi, G.; Peres, B.; Lightbody, M.; Gauthier, C.;

Ozvegy-Laczka, C.; Szakacs, G.; Sarkadi, B.; Creczynski-Pasa, T. B.; Boumendjel, A.; Di Pietro, A. J. Med. Chem. 2013, 56(24), 98499860.

24. Gaspar, A.; Silva, T.; Anez, M. Y.; Vina, D.; Orallo, F.; Ortuso, F.; Uriarte, E.; Alcaro, S.; Borges, F. J. Med. Chem. 2011, 54(14), 51655173.
25. Andrews, S. P.; Mason, J. S.; Hurrell, E.; Congreve, M. Hide Affiliations

26. Kang, W.; Du, X.; Wang, L.; Hu, L.; Dong, Y.; Bian, Y.; Li, Y. Chin. J. Chem. 2013, 31(10), 1305-1314.

27. Garg, N.; Chandra, T.; Archana; Jain, A. B.; Kumar, A. Eur. J. Med. Chem. 2010, 45(4), 1529-1535.

28. Ameta, K. L.; Rathore N. S.; Kumar, B. J. Serb. Chem. Soc. 2012, 77(6), 725-731.

29. Ayral, E.; Gloanec, P.; Berge, G.; de Nanteuil, G.; Mennecier, P.; Rupin, A.;Verbeuren, T. J.; Fulcrand, P.; Martinez, J.; Hernandez, J. F. Bioorg. Med. Chem. Lett. 2009, 19(5), 13861391.

30. Tran, P. V.; Bymaster, F. P.; McNamara, R. K.; Potter, W. Z. J. Clin. Psychopharmacol. 2003, 23(1), 78-86.

31. Sauzem, P. D.; Machado, P. M.; Rubin, A.; Sant'Anna, G. S.; Faber, H. B.; De Souza, A. H.; Mello, C. F.; Beck, P.; Burrow, R. A.; Bonacorso, H. G.; Zanatta, N.;

Martins, M. A. P. Eur. J. Med. Chem. 2008, 43(6), 1237-1247.

32. Cunico, W.; Cechinel, C. A.; Bonacorso, H. G.; Martins, M. A. P.; Zanatta, N.; De

Souza, M. V. N.; Freitas, I. O.; Soares, R. P. P.; Krettli, A. U. Bioorg. Med. Chem. Lett. 2006, 16(3), 649-653.

33. Ali, M. A.; Shaharyar, M. and Siddiqui, A. A., Eur. J. Med. Chem. 2007, 42(2), 268-275.

34. Johnson, M.; Younglove, B.; Lee, L.; LeBlanc, R.; Holt, H.; Jr Hills, P.; Mackay, H.; Brown, T.; Mooberry, S. L.; Lee, M. Bioorg. Med. Chem. Lett. 2007, 17(21), 5897-5901. 